\title{
Patient-Specific QA of Spot-Scanning Proton Beams Using Radiochromic Film
}

\author{
Maria F. Chan1, Chin-Cheng Chen², Chengyu Shi' ${ }^{1}$, Jingdong Li', Xiaoli Tang1, Xiang Li', Dennis Mah ${ }^{2}$ \\ ${ }^{1}$ Dept. of Medical Physics, Memorial Sloan Kettering Cancer Center, New York, NY, USA \\ ${ }^{2}$ Dept. of Radiation Physics, ProCure Proton Center, Somerset, NJ, USA \\ Email: chanm@mskcc.org
}

How to cite this paper: Chan, M.F., Chen, C.-C., Shi, C.Y., Li, J.D., Tang, X.L., Li, X. and Mah, D. (2017) Patient-Specific QA of Spot-Scanning Proton Beams Using Radiochromic Film. International Journal of Medical Physics, Clinical Engineering and Radiation Oncology, 6, 111-123.

https://doi.org/10.4236/ijmpcero.2017.62011

Received: February 12, 2017

Accepted: May 13, 2017

Published: May 16, 2017

Copyright $\odot 2017$ by authors and Scientific Research Publishing Inc. This work is licensed under the Creative Commons Attribution International License (CC BY 4.0).

http://creativecommons.org/licenses/by/4.0/

\begin{abstract}
Radiochromic film for spot-scanning QA provides high spatial resolution and efficiency gains from one-shot irradiation for multiple depths. However, calibration can be a tedious procedure which may limit widespread use. Moreover, since there may be an energy dependence, which manifests as a depth dependence, this may require additional measurements for each patient. We present a one-scan protocol to simplify the procedure. A calibration using an EBT3 film, exposed by a 6-level step-wedge plan on a Proteus ${ }^{\mathbb{B}}$ PLUS proton system (IBA, Belgium), was performed at depths of 18,20,24 cm using Plastic Water $^{\circledR}$ (CIRS, Norfolk, VA). The calibration doses ranged from $65-250 \mathrm{cGy}$ (RBE) (relative biological effectiveness) for proton energies of $170-200 \mathrm{MeV}$. A clinical prostate + nodes plan was used for validation. The planar doses at selected depths were measured with EBT3 films and analyzed using one-scan protocol (one-scan digitization of QA film and at least one film exposed to a known dose). The gamma passing rates, dose-difference maps, and profiles of 2D planar doses measured with EBT3 film and IBA MatriXX-PT, versus the RayStation TPS calculations were analyzed and compared. The EBT3 film measurement results matched well with the TPS calculation data with an average passing rate of $\sim 95 \%$ for $2 \% / 2 \mathrm{~mm}$ and slightly lower passing rates were obtained from an ion chamber array detector. We were able to demonstrate that the use of a proton step-wedge provided clinically acceptable results and minimized variations between film-scanner orientation, inter-scan, and scanning conditions. Furthermore, for relative dosimetry (calibration is not done at the time of experiment), it could be derived from no more than two films exposed to known doses (one could be zero) for rescaling the master calibration curve at each depth. The sensitivity of the calibration to depth variations has been explored. One-scan protocol results appear to be comparable to that of the ion chamber array detector. The use of a proton step-wedge for calibration of EBT3 film potentially increases efficiency in patient-specific QA of proton beams.
\end{abstract}




\section{Keywords}

Proton Therapy, Patient-Specific QA, Gaf Chromic EBT3, Film Dosimetry

\section{Introduction}

Owing to its dosimetric characteristics, particularly finite range, proton therapy provides a potentially favorable treatment option for many cancer patients [1] [2]. Protons show an increasing energy deposition with penetration distance leading to a maximum dose (the Bragg peak) near the end of range of the proton beam. A weighted sum of individual Bragg peaks of different energies can be used to generate a uniform dose plateau or spread-out Bragg peak (SOBP), which in turn, can be overlaid over the target during treatment planning. The SOBP is characterized by its range and width, or modulation [3]. Delivery of an SOBP has used different techniques including most commonly passive scattering [4] [5] as well as uniform scanning [6] [7].

More recently, spot scanning or pencil beam scanning (PBS) has emerged as a delivery technique [8] [9]. Unlike scattered beams, in which the dose is shaped by a selection of the appropriate range and modulation and shaped with a customized compensator and aperture, in spot scanning, the beam is delivered and shaped by scanning individually weighted spots to a radiological depth. The energy is then changed and the next layer is scanned. Analogous to x-ray treatment planning, scatter beams are forward planned in $3 \mathrm{D}$, whereas spot scanning is inversely planned [10] [11].

The current standard of patient-specific QA for spot scanning proton therapy is to perform measurements using a chamber array at different depths followed by a $2 \mathrm{D}$ or $3 \mathrm{D}$ gamma analysis [12] [13]. Radiochromic film for spot scanning QA provides high spatial resolution and efficiency gains from one-shot irradiation at multiple depths. The use of radiochromic film for protons has been reported in a variety of investigations [14]-[22]. However, calibration can be a tedious procedure that may reduce widespread use. Moreover, since there may be an energy dependence, which manifests as a depth dependence, this may require additional measurements for each patient [23]. We applied the one-scan protocol [24] to simplify the procedure. Briefly, this approach requires only a single calibration exposure to a known dose of one film and a single scan of the QA film thereby vastly simplifying what the previous approach of irradiating multiple films for calibration each time. This study aims to investigate the effectiveness of one-scan protocol on proton dosimetry, focusing attention on its application to spot scanning patient-specific QA.

\section{Materials and Methods}

Irradiations were performed using a spot scanning beam delivery on a Proteus ${ }^{\circledR}$ PLUS proton system (IBA, Belgium) at the ProCure Proton Therapy Center in Somerset, NJ. Typical energy ranges from the nozzle vary from $\sim 100$ to 
$230 \mathrm{MeV}$, however, much lower effective energies can be obtained for treating superficial depths using a range shifter. The details of the beam delivery system are described in a separate study [25].

The EBT3 films (lot 02181401, Ashland, Bridgewater, NJ) were irradiated in Plastic Water ${ }^{\circledR}$ (CIRS, Norfolk, VA) at depths of 18,20 , and $23 \mathrm{~cm}$ by a 6 -level step-wedge plan. Each film sheet was sandwiched between Plastic Water slabs perpendicular to the beam direction. The step-wedge plan consists of 17 different spot energy layers (145 - $198 \mathrm{MeV}$ ) and a different number of spots and weights (Table 1).

The goal of selecting calibration doses is to equilibrate uncertainty in high and low dose areas. The equi-color dose scheme ensures the high and low dose regions are equally well approximated for EBT3 film. The calibration doses of 65, $91,128,179$, and $250 \mathrm{cGy}(\mathrm{RBE})$ (relative biological effectiveness) were based on such scheme. All treatment plans were generated in the RayStation (RaySearch Laboratories, Sweden) including the calibration step-wedge plan (Figure 1). Dose distributions measured with EBT3 film were also compared with those obtained with MatriXX-PT (IBA Dosimetry, Germany). The responses of the exposed films were evaluated by a flatbed Epson 10000XL scanner at 72 dpi resolution.

A clinical treatment plan for prostate with seminal-vesicles and pelvis-nodal

Table 1. The proton energy layers for the calibration. The films were placed at $18,20,23$ $\mathrm{cm}$ depth Plastic Water ${ }^{\circledR}$. Only protons with energy $>160 \mathrm{MeV}$ deposited dose to the films.

\begin{tabular}{|c|c|c|c|c|c|}
\hline \multirow{2}{*}{ No. } & \multirow{2}{*}{ Energy $[\mathrm{MeV}]$} & \multirow{2}{*}{ Relative Weight [\%] } & \multirow{2}{*}{ Number of Spots } & \multicolumn{2}{|c|}{ Spot Weight $[\mathrm{MU} / \mathrm{fx}]$} \\
\hline & & & & Min & $\operatorname{Max}$ \\
\hline 1 & 197.81 & 33.96 & 3452 & 0.0875 & 1.7864 \\
\hline 2 & 194.37 & 11.73 & 3404 & 0.0224 & 1.1532 \\
\hline 3 & 190.97 & 9.01 & 3640 & 0.0226 & 1.0941 \\
\hline 4 & 187.56 & 6.56 & 3589 & 0.0225 & 0.6605 \\
\hline 5 & 184.17 & 5.58 & 3476 & 0.0222 & 0.8894 \\
\hline 6 & 180.77 & 4.70 & 3415 & 0.0223 & 0.6062 \\
\hline 7 & 177.40 & 4.03 & 3371 & 0.0224 & 0.5153 \\
\hline 8 & 174.06 & 3.41 & 3159 & 0.0222 & 0.4507 \\
\hline 9 & 170.76 & 2.92 & 2834 & 0.0225 & 0.4004 \\
\hline 10 & 167.46 & 2.62 & 2609 & 0.0226 & 0.3700 \\
\hline 11 & 164.17 & 2.25 & 2369 & 0.0225 & 0.2678 \\
\hline 12 & 160.87 & 2.61 & 2069 & 0.0231 & 0.5404 \\
\hline 13 & 157.59 & 2.20 & 2023 & 0.0230 & 0.4947 \\
\hline 14 & 154.34 & 1.96 & 1923 & 0.0229 & 0.4417 \\
\hline 15 & 151.16 & 1.82 & 1776 & 0.0230 & 0.2653 \\
\hline 16 & 148.03 & 1.44 & 1259 & 0.0230 & 0.4709 \\
\hline 17 & 144.99 & 3.20 & 3583 & 0.0246 & 0.0461 \\
\hline
\end{tabular}




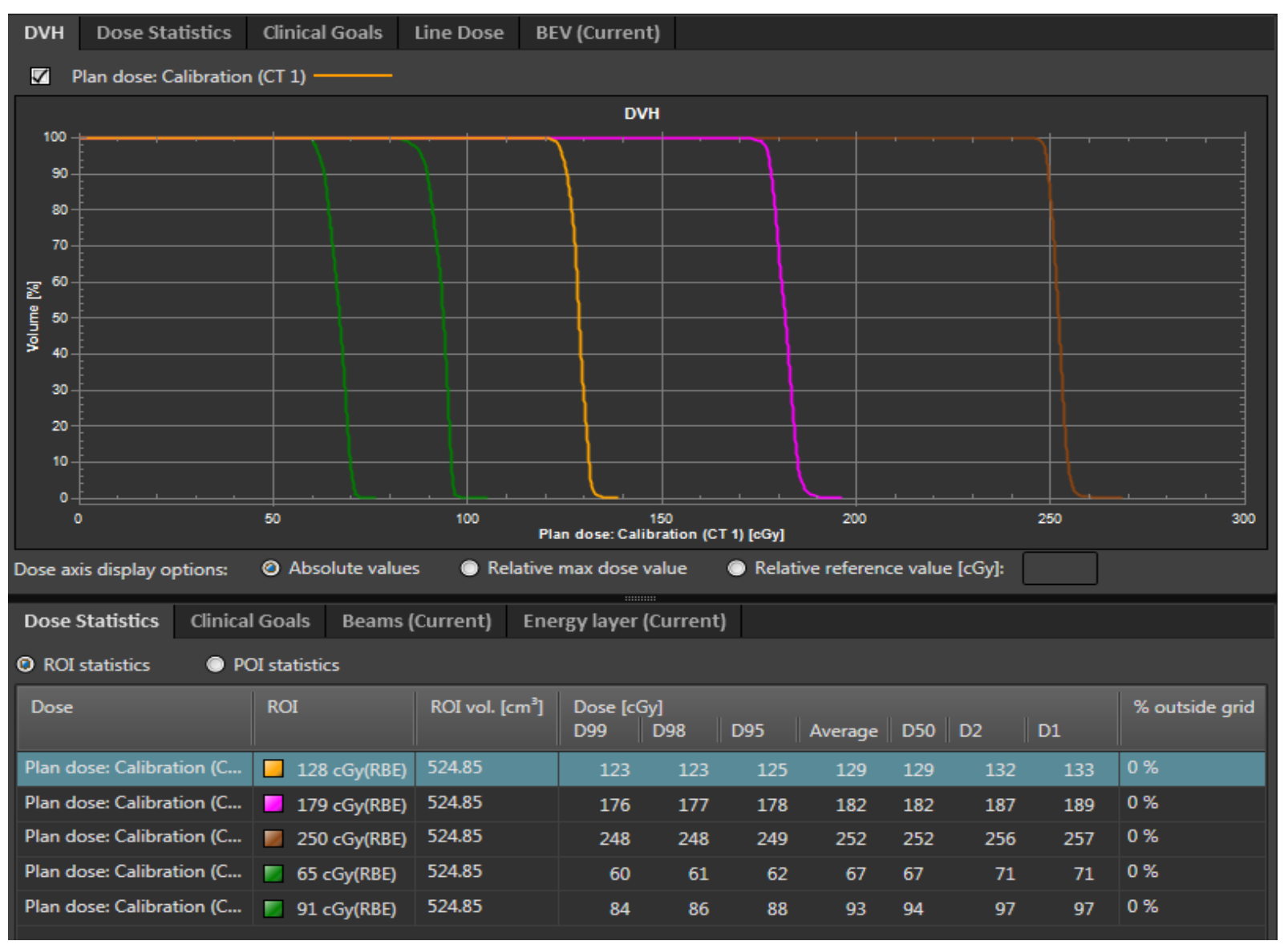

Figure 1. Screenshot of the treatment plan of the step-wedge calibration.

involvement was used for testing (Figure 2). The planar doses at different depths were measured with EBT3 film and the MatriXX-PT. Calibration films were irradiated at 18,20 , and $23 \mathrm{~cm}$ while the chamber array was irradiated at nearly identical depths of 18, 20 and $24 \mathrm{~cm}$. The LET dependence of radiochromic films has been thoroughly investigated [6] [18] [22] [26]-[33] and it has been established that the effect is highest for lower $(<11 \mathrm{MeV})$ proton energies [28]. However, for spot scanning beams, there may be a larger distribution of energies used to irradiate the PTV which could make accounting for LET corrections quite variable. To assess the depth (energy) dependence, we performed gamma analysis using calibration films at different depths. The films were analyzed using One-scan protocol (one-scan digitization of QA film and at least one film exposed to a known dose). We used the $180 \mathrm{cGy}(\mathrm{RBE})$ calibration dose and the zero dose to rescale the dose response of the patient film. This is analogous to the absolute dose calibration for diode array or ion chamber array detectors before the patient-specific QA takes place. The gamma passing rates, dose-difference maps, and profiles of 2D planar doses measured with EBT3 film and IBA MatriXX-PT, versus the RayStation TPS calculations were analyzed and compared.

\section{Results}

The dose-response data could be fit to a set of related rational functions leading 


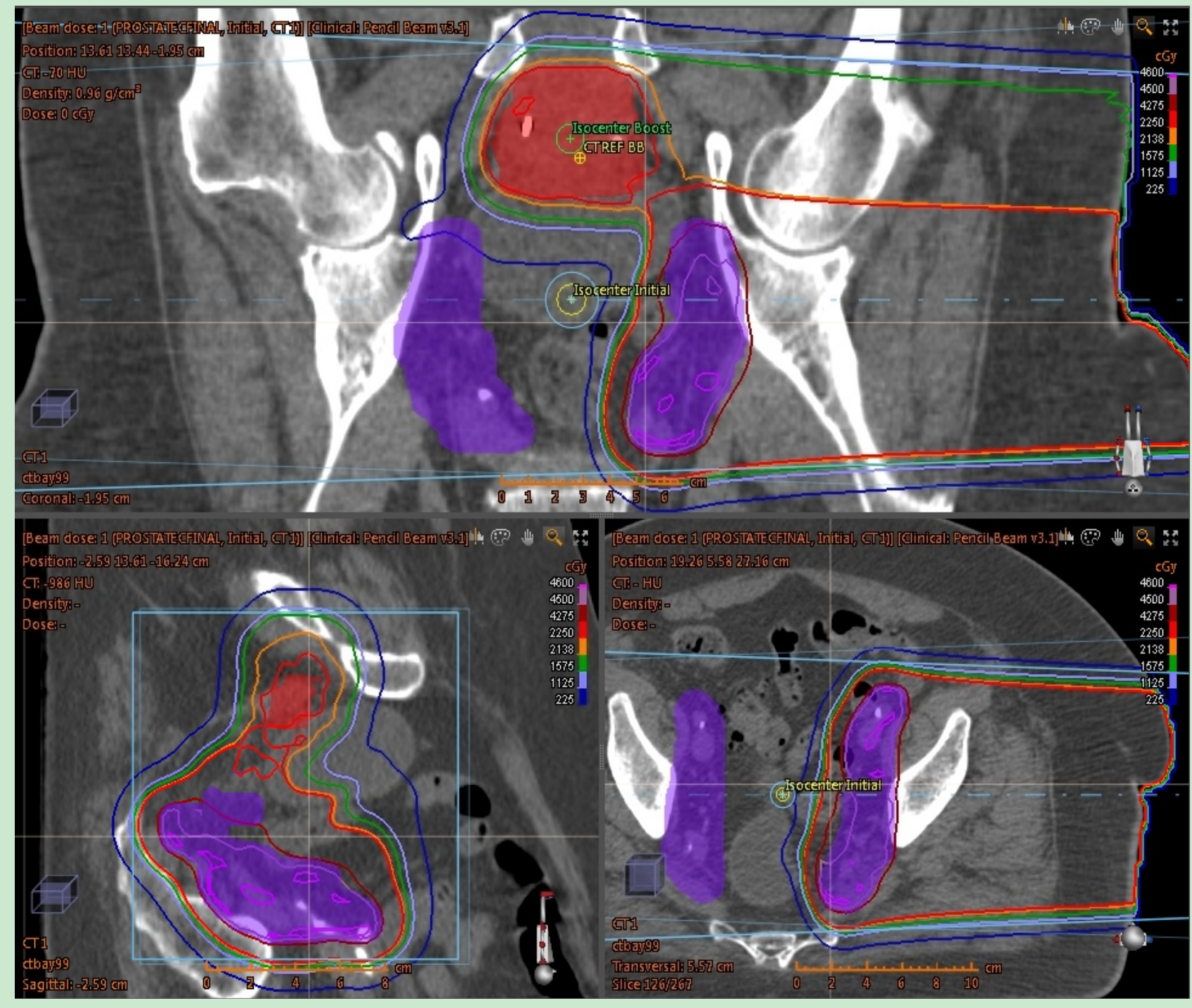

Figure 2. Three orthogonal dose distributions with target doses of $90 \mathrm{cGy}(\mathrm{RBE})$ (red), $180 \mathrm{cGy}(\mathrm{RBE})$ (purple) treated laterally; coronal view (upper center), sagittal view (lower left), transverse view (lower right).

to the description of a generic calibration curve for a given film lot [34]. One generic calibration curve was generated using the scanned step-wedge film at each depth (Figure 3). For comparison of MatriXX-PT, a similar dose map and step-level were used. The dose-response data for a specific film lot, scanner, and scanning conditions could be derived from two films exposed to known doses including zero dose. For relative dosimetry when the calibration is not done at the time of patient QA, two-point rescaling could be applied to rebuild the master calibration curve at each depth. Again, the selection of the two-point doses (one of them could be zero) depends on the maximum dose level on the plane of patient QA film. These calibration films must be exposed at the same time as the QA film in order to be compliant with the One-scan protocol. Each QA film was scanned together with an exposed reference film (irradiated within minutes of the QA films) and a piece of unexposed film. 

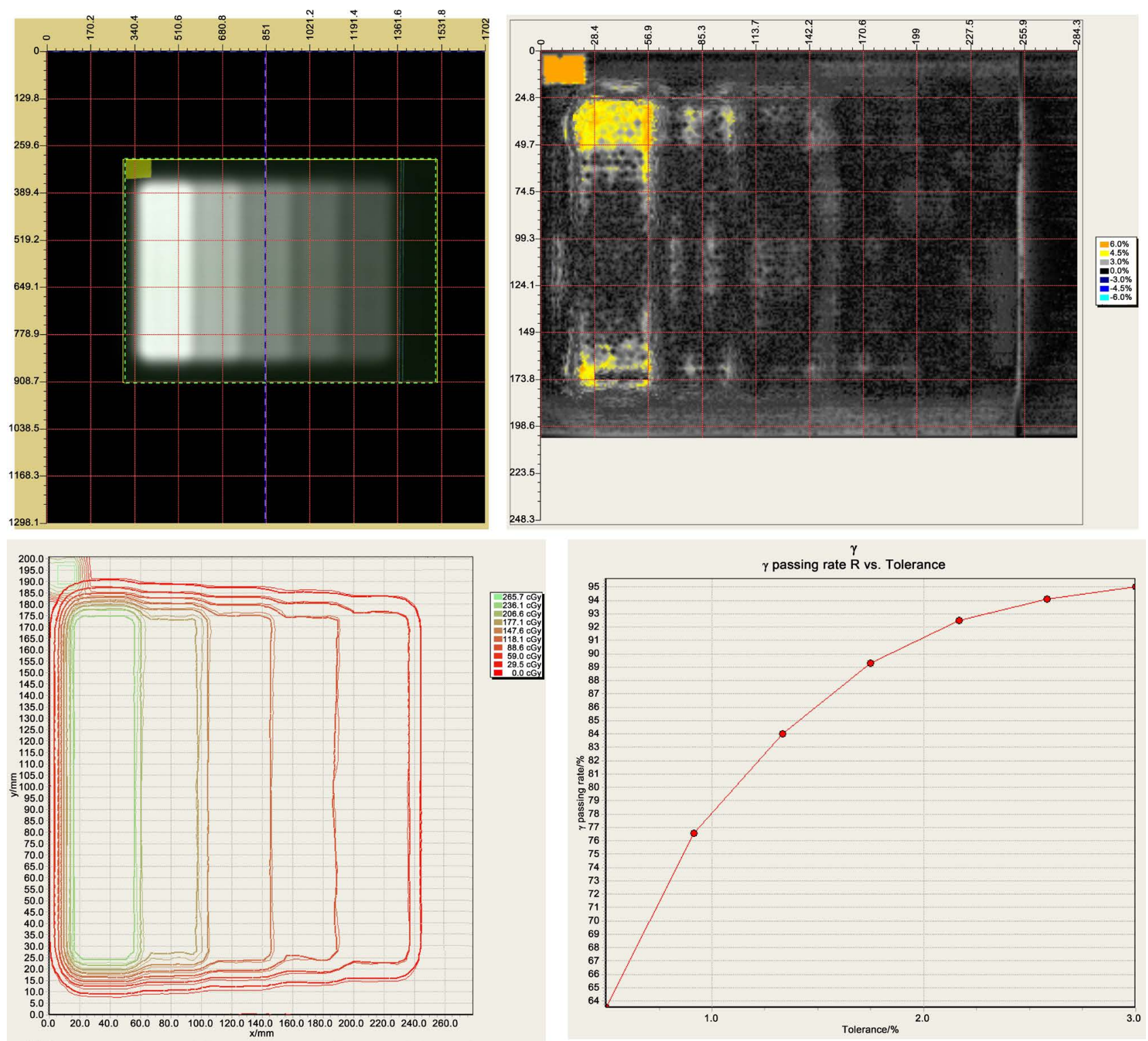

Figure 3. Screenshots of scanned step-wedge film in FilmQA Pro: scanned film image (upper left); isodose lines (lower left); dose difference map (upper right); passing rates as function of gamma (lower right).

Using the gamma test criteria of $3 \% / 3 \mathrm{~mm}, 3 \% / 2 \mathrm{~mm}, 2 \% / 2 \mathrm{~mm}$ to evaluate the digital image consisting of the patient film, one calibration film, and one unexposed film for calculation and analysis, similar passing rates were obtained between EBT3 film and MatriXX-PT except for the largest discrepancy with the $2 \% / 2 \mathrm{~mm}$ criteria at the shallowest depth $(\mathrm{d}=18 \mathrm{~cm})$ in the prostate case. Figure 4(a), Figure 4(b) show the isodoses overlay and dose difference map of film and TPS data. Figure 5(a) shows the vertical dose profile of the sagittal view of dose distribution of the prostate plan. Figure 5(b) shows the gamma passing rate as a function of dose difference (DD) and distance-to-agreement (DTA). The EBT3 film measurement results matched well with the TPS calculation data with an average passing rate of $\sim 95 \%$ for $2 \% / 2 \mathrm{~mm}$ and slightly lower passing rates were obtained from an ion chamber array detector (Table 2). 


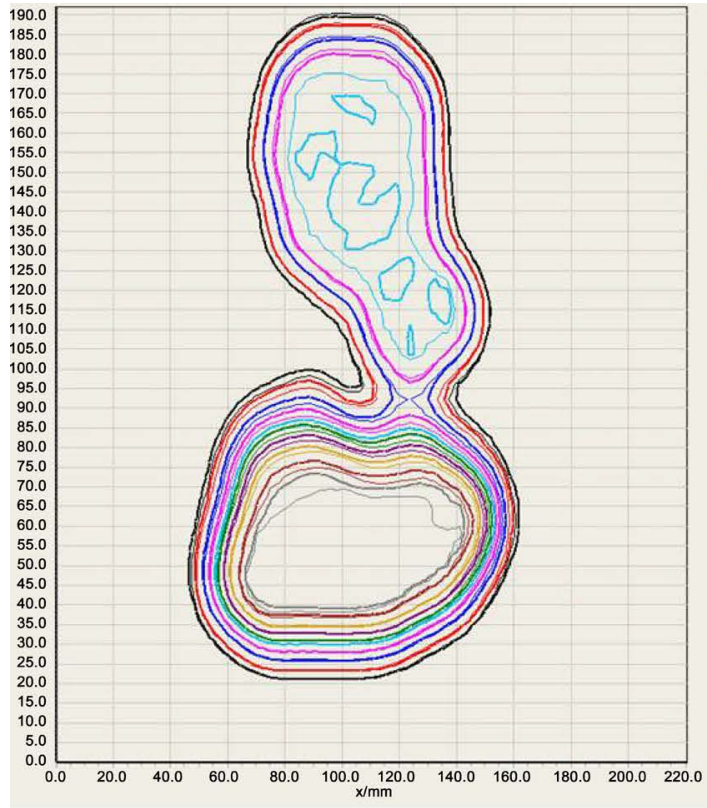

(a)
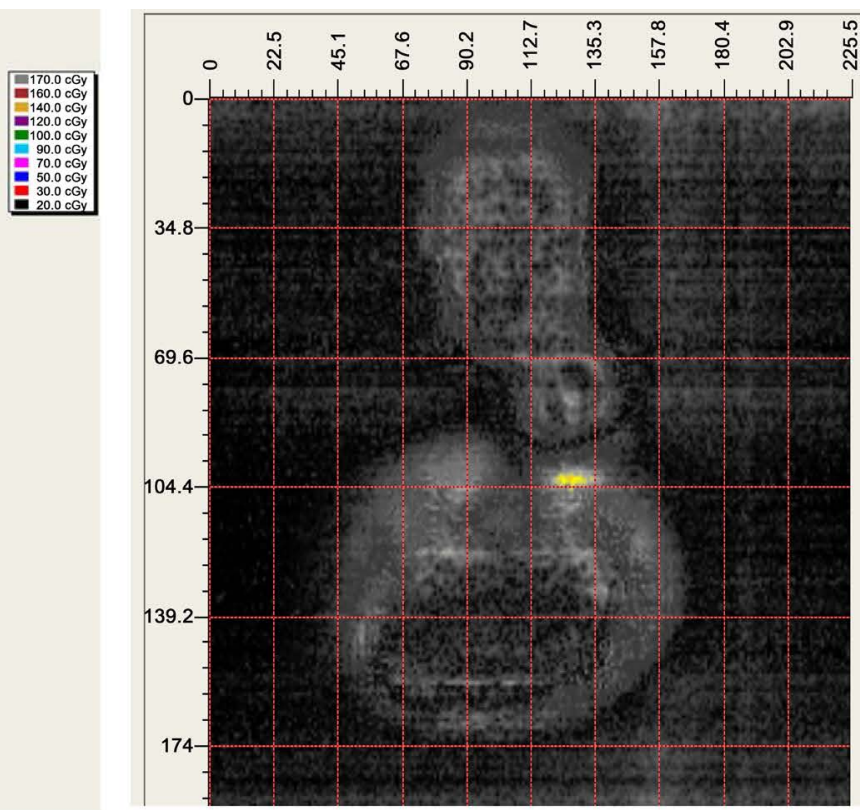

(b)

Figure 4. (a) Sagittal plane isodoses overlay; (b) Dose difference map.

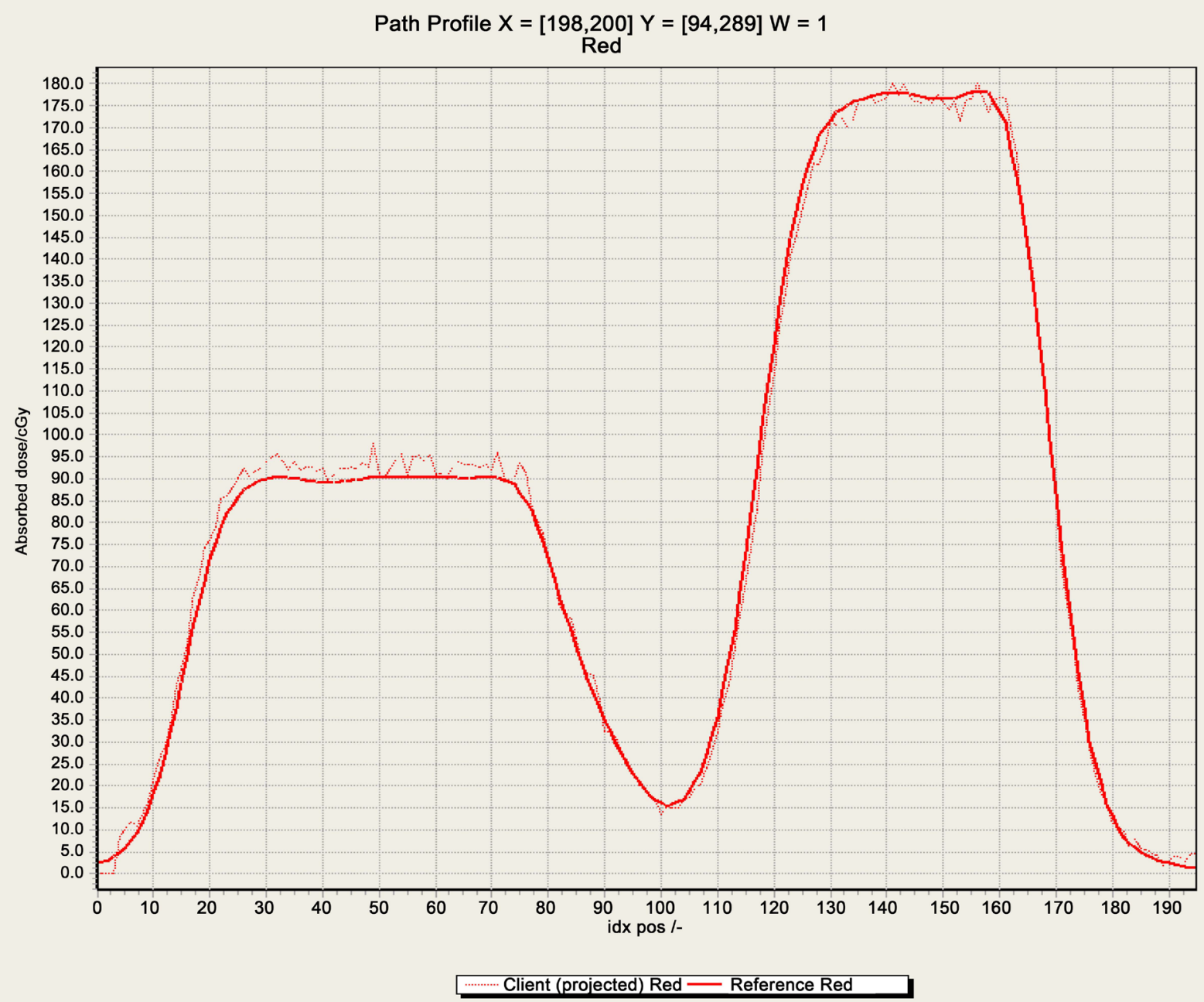

(a) 


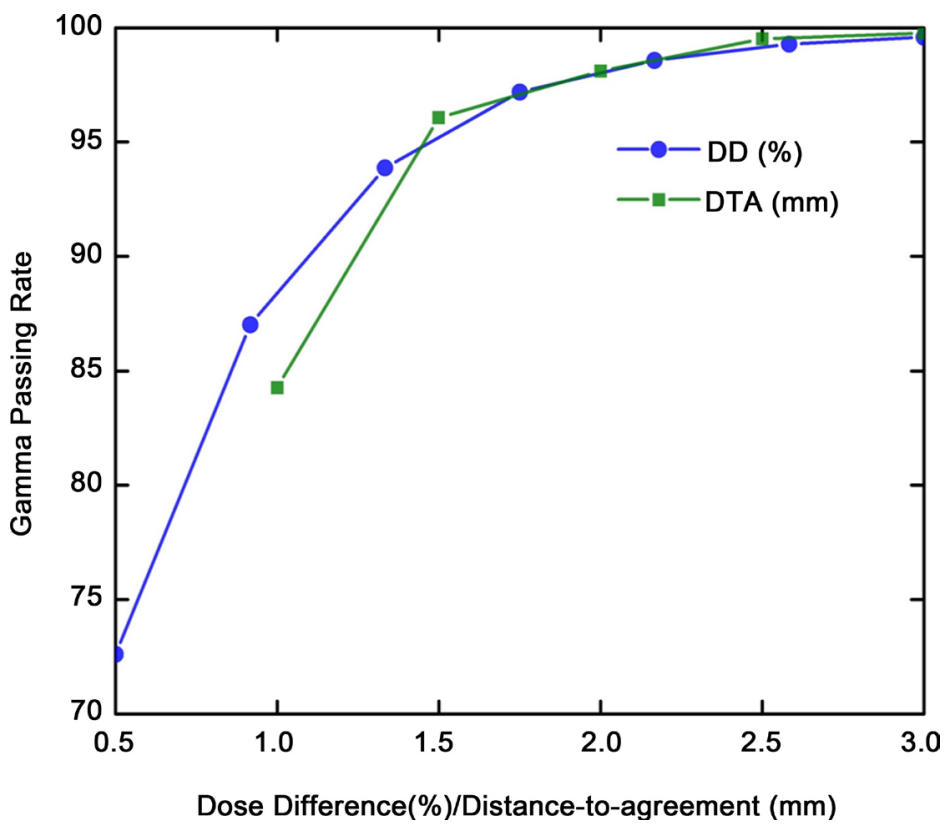

(b)

Figure 5. (a) Vertical dose profile of the sagittal distribution; (b) Gamma passing rate as a function of dose.

Table 2. Gamma passing rates of dose comparison between film/TPS and MatriXX/TPS.

\begin{tabular}{|c|c|c|c|c|c|c|c|}
\hline \multirow{3}{*}{ Proton Beam } & \multirow{3}{*}{ Depth $(\mathrm{cm})$} & \multicolumn{6}{|c|}{ Criteria of Gamma Evaluation } \\
\hline & & \multicolumn{2}{|c|}{$3 \%, 3 \mathrm{~mm}$ DTA } & \multicolumn{2}{|c|}{$3 \%, 2 \mathrm{~mm}$ DTA } & \multicolumn{2}{|c|}{$2 \%, 2 \mathrm{~mm}$ DTA } \\
\hline & & EBT3 & MatriXX & EBT3 & MatriXX & EBT3 & MatriXX \\
\hline \multirow{3}{*}{ Prostate + SV + Pelvis Nodes } & 18 & $99.2 \%$ & $98.7 \%$ & $98.6 \%$ & $96.1 \%$ & $94.3 \%$ & $89.5 \%$ \\
\hline & 20 & $98.5 \%$ & $99.1 \%$ & $98.4 \%$ & $97.4 \%$ & $96.1 \%$ & $93.5 \%$ \\
\hline & 23 & $97.9 \%$ & $99.5 \%$ & $97.5 \%$ & $98.4 \%$ & $96.7 \%$ & $95.9 \%$ \\
\hline
\end{tabular}

Note: The MatriXX-PT was taken at $24 \mathrm{~cm}$ instead of $23 \mathrm{~cm}$.

Table 3. Highest passing rate with the corresponding calibration curve at each depth.

\begin{tabular}{ccc}
\hline Passing Rate $(3 \%, 3 \mathrm{~mm}$ DTA $)$ & Calibration $(\mathrm{d}=18 \mathrm{~cm})$ & Calibration $(\mathrm{d}=20 \mathrm{~cm})$ \\
\hline QA Film $(\mathrm{d}=18 \mathrm{~cm})$ & $99.2 \%$ & $96.0 \%$ \\
QA Film $(\mathrm{d}=20 \mathrm{~cm})$ & $95.5 \%$ & $98.5 \%$ \\
QA Film $(\mathrm{d}=23 \mathrm{~cm})$ & $90.0 \%$ & $97.3 \%$ \\
\hline
\end{tabular}

To explore the depth dependence, we have applied different combinations of calibration curves and patient QA films at 3 different depths to assess the effect. Table 3 demonstrates the highest passing rate occurred when using calibration and QA film at the same depth; therefore it suggests having a different calibration function at a different depth.

\section{Discussion}

Radiochromic film dosimetry in low-energy proton or ion beams requires a cor- 
rection of the LET dependent film response [35] [36]. Radiochromic film can be used as a reference dosimeter for biomedical experiments with low-energy proton beams if appropriate LET corrections are applied [36]. There are numerous researchers reported that under-response of radiochromic film in the presence of proton SOBPs which could be defined as "quenching effect" of the dosimeter in the region [26]-[33] [35] [36] [37]. "Quenching" is said to occur when, for a given physical dose, a dosimeter not limited to radiochromic film gives a lower output reading for radiation with high LET than for the same dose of low LET radiation. The effects are particularly noticeable for measurements made at the Bragg peak in proton treatments [38]. Quenching of dosimeter response is a major issue in the measurement of the radiation dose from proton and other ion beams. The quenching effect of EBT3 film is the highest at the lowest initial energy of the clinical beams, a phenomenon related to the corresponding higher LET in the film sensitive layer [35]. A possible reason for this is attributed to the shape and arrangement of the monomer particles being different in the active components of EBT. It is suggested that the polymer yield factor is the dominant factor causing the LET quenching effect [29].

Table 3 demonstrates the depth dependence of the calibration film. There is clearly dependence and for optimal results, a calibration film should be generated at the same depth as the measurement. This finding is in agreement with previous studies for depth calibration [39] [40] or at the center of the SOBP [6] [17] [19]. However, the dependence for different ranges and modulations requires further investigation.

Another alternative method reported for calibration rather than multiple measurements at different depths were reported by Vadrucci et al. The film is positioned at the entrance of a $5 \mathrm{MeV}$ proton beam with the film data acquired from a readout system, taking average data over a region of interest of uniform dose at the beam center for each film and plot against the known dose [30]. The particle energy of proton beam is variable with depth and could be losing energy continuously (lowest at Bragg peak and highest effect in depth dependence). The energy response is also dependent on the position of the Bragg peak [6].

The film orientation relative to the proton beam direction also plays an important role in dosimetry due to radiation interaction's causing polymerization in radiochromic film [7]. The parallel orientation was found to be superior as one can get an entire 2D data set from one single film. When using a single film piece positioned parallel to the proton beam axis, it is recommended to tilt the film plane at different degrees $\left(1^{\circ}-5^{\circ}\right)$ from the proton beam axis or the film can be kept in water [6] [18]. The film had sufficient width so that water in the film would not cause additional perturbation. In our work, we have positioned the EBT3 film perpendicular to the beam axis, calibrated the step-wedge film with the mixed proton energies at each depth, used one-scan protocol for film analysis to eliminate the inter-scan variability and corrected for post-exposure density growth. The film results are comparable with the ion chamber array measurements as respect to the TPS calculations. 


\section{Conclusion}

The use of a proton step-wedge for calibration of EBT3 film appears to increase the efficiency. The sensitivity of the calibration to depth variations has been explored. However, the step-wedge approach should be limited to film dosimetry at the range which is not at the distal edge of SOBP. The LET dependence of EBT3 needs to be incorporated in the process to improve the accuracy of film dosimetry at lower proton energies or when the film is parallel to the beam axis.

\section{Acknowledgements}

This research was funded in part through the NIH/NCI Cancer Center Support Grant P30 CA008748. Maria Chan has a research grant from Ashland Inc., the manufacturer of the GafChromic film.

\section{References}

[1] Paganetti, H. and Bortfeld, T. (2006) Proton Beam Radiotherapy-The State of the Art. In: Schlegel, T.B.W. and Grosu, A.L., Eds., New Technologies in Radiation Oncology, Springer-Verlag, Berlin, 345-363. https://doi.org/10.1007/3-540-29999-8_27

[2] Das, I.J. and Paganett, H. (2015) Principles and Practice of Proton Beam Therapy, AAPM Monograph No. 37. Medical Physics Publishing Inc., Madison.

[3] International Commission on Radiation Units and Measurements (2007) Prescribing, Recording, and Reporting Proton-Beam Therapy (ICRU Report 78). Journal of the International Commission on Radiation Units, 7, No. 2.

[4] Arjomandy, B., Sahoo, N., Zhu, X.R., et al. (2009) An Overview of the Comprehensive Proton Therapy Machine Quality Assurance Procedures Implemented at The University of Texas M. D. Anderson Cancer Center Proton Therapy CenterHouston. Medical Physics, 36, 2269-2282. https://doi.org/10.1118/1.3120288

[5] Sahoo, N., Sawakuchi, G.O., Gillin, M.T. and Zhu, X.R. (2016) Radiation Dosimetry of Proton Beams, Chapter 6. In: Rath, A.K. and Sahoo, N., Eds., Particle Radiotherapy, Springer India, New Delhi, 77-94. https://doi.org/10.1007/978-81-322-2622-2_6

[6] Zhao, L. and Das, I.J. (2010) Gafchromic EBT Film Dosimetry in Proton Beams. Physics in Medicine and Biology, 55, N291-N301. https://doi.org/10.1088/0031-9155/55/10/N04

[7] Farr, J.B., Mascia, A.E., His, W.C., et al. (2008) Clinical Characterization of a Proton Beam Continuous Uniform Scanning System with Dose Layer Stacking. Medical Physics, 35, 4945-4954. https://doi.org/10.1118/1.2982248

[8] Gillin, M.T., Sahoo, N., Bues, M., et al. (2010) Commissioning of the Discrete Spot Scanning Proton Beam Delivery System at The University of Texas MD Anderson Cancer Center, Proton Therapy Center, Houston. Medical Physic, 37, 154-163. https://doi.org/10.1118/1.3259742

[9] Schwarz, M., Algranati, C., Widesott, L., Farace, P., et al. (2016) Clinical Pencil Beam Scanning: Present and Future Practices. In: Rath, A.K. and Sahoo, N., Eds., Particle Radiotherapy, Springer-Verlag, Berlin, 95-110. https://doi.org/10.1007/978-81-322-2622-2_7

[10] Lomax, A.J., Boehringer, T., Coray, A., et al. (2001) Intensity Modulated Proton Therapy: A Clinical Example. Medical Physics, 28, 317-324.

https://doi.org/10.1118/1.1350587 
[11] Dietlicher, I., Casiraghi, M., Ares, C., et al. (2014) The Effect of Surgical Titanium Rods on Proton Therapy Delivered for Cervical Bone Tumors: Experimental Validation Using an Anthropomorphic Phantom. Physics in Medicine and Biology, 59, 7181-7194. https://doi.org/10.1088/0031-9155/59/23/7181

[12] Mackin, D., Li, Y., Taylor, M.B., et al. (2013) Improving Spot-Scanning Proton Therapy Patient Specific Quality Assurance with HPlusQA, a Second-Check Dose Calculation Engine. Medical Physics, 40, Article ID: 121708.

https://doi.org/10.1118/1.4828775

[13] Mackin, D., Zhu, X.R., Poenisch, F., et al. (2014) Spot-Scanning Proton Therapy Patient-Specific Quality Assurance: Results from 309 Treatment Plans. International Journal of Particle Therapy, 1, 711-720. https://doi.org/10.14338/IJPT-14-00017.1

[14] Hui, C.K., Robertson, D., Alsanea, F. and Beddar, S. (2015) Fast Range Measurement of Spot Scanning Proton Beams Using a Volumetric Liquid Scintillator Detector. Journal of Biomedical Physics and Engineering, 1, Article ID: 025205. https://doi.org/10.1088/2057-1976/1/2/025204

[15] Molinelli, S., Mairani, A., Mirandola, A., et al. (2013) Dosimetric Accuracy Assessment of a Treatment Plan Verification System for Scanned Proton Beam Radiotherapy: One-Year Experimental Results and Monte Carlo Analysis of the Involved Uncertainties. Physics in Medicine and Biology, 58, 3837-3847. https://doi.org/10.1088/0031-9155/58/11/3837

[16] Zhu, X.R., Poenisch, F., Song, X.F., et al. (2011) Patient-Specific Quality Assurance for Prostate Cancer Patients Receiving Spot Scanning Proton Therapy Using SingleField Uniform Dose. International Journal of Radiation Oncology, Biology, Physics, 81, 552-559. https://doi.org/10.1016/j.ijrobp.2010.11.071

[17] Arjomandy, B., Tailor, R., Anand, A., et al. (2010) Energy Dependence and Dose Response of Gafchromic EBT2 Film over a Wide Range of Photon, Electron, and Proton Beam Energies. Medical Physics, 37, 571-576.

https://doi.org/10.1118/1.3373523

[18] Angellier, G., Gautier, M. and Herault, J. (2011) Radiochromic EBT2 Film Dosimetry for Low-Energy Proton Therapy. Medical Physics, 38, 6171-6177. https://doi.org/10.1118/1.3654161

[19] Arjomandy, B., Tailor, R., Zhao, L., et al. (2012) EBT2 Film as a Depth-Dose Measurement Tool for Radiotherapy Beams over a Wide Range of Energies and Modalities. Medical Physics, 39, 912-921. https://doi.org/10.1118/1.3678989

[20] Sorriaux, J., Kacperek, A., Rossomme, S., et al. (2013) Evaluation of Gafchromic ${ }^{\infty}$ EBT3 Films Characteristics in Therapy Photon, Electron and Proton Beams. Physica Medica, 29, 599-606. https://doi.org/10.1016/j.ejmp.2012.10.001

[21] Carnicer, A., Angellier, G., Gerard, A., et al. (2013) Development and Validation of Radiochromic Film Dosimetry and Monte Carlo Simulation Tools for Acquisition of Absolute, High-Spatial Resolution Longitudinal Dose Distributions in Ocular Proton Therapy. Radiation Measurements, 59, 225-232. https://doi.org/10.1016/j.radmeas.2013.06.011

[22] Gambarini, G., Regazzoni, V., Artuso, E., Giove, D., Mirandola, A. and Ciocca, M. (2015) Measurements of 2D Distributions of Absorbed Dose in Proton Therapy with Gafchromic EBT3 Films. Applied Radiation and Isotopes, 104, 192-196. https://doi.org/10.1016/j.apradiso.2015.06.036

[23] Chan, M.F., Chen, C.C., Li, J., et al. (2016) One-Scan Protocol: Verifying the Delivery of Spot-Scanning Proton Beam. Medical Physics, 43, 3501. https://doi.org/10.1118/1.4956310

[24] Lewis, D., Micke, A., Yu, X. and Chan, M.F. (2012) An Efficient Protocol for Radio- 
chromic Film Dosimetry Combining Calibration and Measurement in a Single Scan. Medical Physics, 39, 6339-6350. https://doi.org/10.1118/1.4754797

[25] Chen, C.C., Chang, C., Moyers, M.F., Gao, M. and Mah, D. (2016) Technical Note: Spot Characteristic Stability for Proton Pencil Beam Scanning. Medical Physics, 43, 777-782. https://doi.org/10.1118/1.4939663

[26] Mayer, R., Lin, L.Y., Fager, M., Douglas, D., McDonough, J. and Carabe, A. (2015) Proposed Linear Energy Transfer Areal Detector for Protons Using Radiochromic Film. Review of Scientific Instruments, 86, Article ID: 044301. https://doi.org/10.1063/1.4917418

[27] Reinhardt, S., Hillbrand, M., Wilkens, J.J. and Assmann, W. (2012) Comparison of Gafchromic EBT2 and EBT3 Films for Clinical Photon and Proton Beams. Medical Physics, 39, 5257-5262. https://doi.org/10.1118/1.4737890

[28] Reinhardt, S., Wurl, M., Greubel, C., et al. (2015) Investigation of EBT2 and EBT3 Films for Proton Dosimetry in the 4-20 MeV Energy Range. Radiation and Environmental Biophysics, 54, 71-79. https://doi.org/10.1007/s00411-014-0581-2

[29] Kirby, D., Green, S., Palmans, H., et al. (2010) LET Dependence of Gafchromic Films and an Ion Chamber in Low Energy Proton Dosimetry. Physics in Medicine and Biology, 55, 417-433. https://doi.org/10.1088/0031-9155/55/2/006

[30] Vadrucci, M., Esposito, G., Ronsivalle, C., et al. (2015) Calibration of GafChromic EBT3 for Absorbed Dose Measurements in $5 \mathrm{MeV}$ Proton Beam and ${ }^{60} \mathrm{Co} \gamma$-Rays. Medical Physics, 42, 4678-4684. https://doi.org/10.1118/1.4926558

[31] Perles, L.A., Mirkovic, D., Anand, A., Titt, U. and Mohan, R. (2013) LET Dependence of the Response of EBT2 Films in Proton Dosimetry Modeled as a Bimolecular Chemical Reaction. Physics in Medicine and Biology, 58, 8477-8491. https://doi.org/10.1088/0031-9155/58/23/8477

[32] Park, S.A., Kwak, W., Yoon, M.G., et al. (2011) Dose Verification of Proton Beam Therapy Using the Gafchromic EBT Film. Radiation Measurements, 46, 717-721. https://doi.org/10.1016/j.radmeas.2011.06.027

[33] Battaglia, M.C., Schardt, D., Espino, J.M., et al. (2016) Dosimetric Response of Radiochromic Films to Protons of Low Energies in the Bragg Peak Region. Physical Review Accelerators and Beams, 19, Article ID: 064701. https://doi.org/10.1103/physrevaccelbeams.19.064701

[34] Chan, M.F., Lewis, D. and Yu, X. (2014) Is It Possible to Publish a Calibration Function for Radiochromic Film? International Journal of Radiation Oncology, Biology, Physics, 3, 25-30. https://doi.org/10.4236/ijmpcero.2014.31005

[35] Castriconi, R., Ciocca, M., Mirandola, A., et al. (2016) Dose-Response of EBT3 Radiochromic Films to Proton and Carbon Ion Clinical Beams. Physics in Medicine and Biology, 62, 377-393. https://doi.org/10.1088/1361-6560/aa5078

[36] Reinhardt, S., Wuerl, M., Greubel, C., et al. (2015) Dependence Correction of Radiochromic Films for Application in Low Energy Proton Irradiation. Medical Physics, 42, 3458. https://doi.org/10.1118/1.4924895

[37] Fiorini, F., Kirby, D., Thompson, J., et al. (2014) Under-Response Correction for EBT3 Films in the Presence of Proton Spread out Bragg Peaks. Physica Medica, 30, 454-461. https://doi.org/10.1016/j.ejmp.2013.12.006

[38] Doran, S.J., Pellicioli, P., Adamovics, J. and Brauer-Krisch, E. (2016) Enhanced Method for Determining the Low-LET Saturation Dose of PRESAGE. Proceedings of the 9th International Conference on 3D Radiation Dosimetry, Galveston, 7-10 November 2016.

[39] Devic, S., Aldelaijan, S., Alrumayan, F., et al. (2013) Radiochromic Film as a Dosi- 
metric Tool for Low Energy Proton Beams. Proceedings of Cyclotrons 2013, Vancouver, 16-20 September 2013, WEPSH007.

[40] Piermattei, A., Miceli, R., Azario, L., et al. (2000) Radiochromic Film Dosimetry of a Low Energy Proton Beam. Medical Physics, 27, 1655-1660.

https://doi.org/10.1118/1.599032

Submit or recommend next manuscript to SCIRP and we will provide best service for you:

Accepting pre-submission inquiries through Email, Facebook, LinkedIn, Twitter, etc. A wide selection of journals (inclusive of 9 subjects, more than 200 journals)

Providing 24-hour high-quality service

User-friendly online submission system

Fair and swift peer-review system

Efficient typesetting and proofreading procedure

Display of the result of downloads and visits, as well as the number of cited articles Maximum dissemination of your research work

Submit your manuscript at: http://papersubmission.scirp.org/

Or contactijmpcero@scirp.org 